\title{
Acetaldehyde as an underestimated risk factor for cancer development: role of genetics in ethanol metabolism
}

\author{
Helmut K. Seitz $\cdot$ Felix Stickel
}

Received: 27 July 2009/Accepted: 23 September 2009/Published online: 22 October 2009

(C) Springer-Verlag 2009

\begin{abstract}
Chronic ethanol consumption is a strong risk factor for the development of certain types of cancer including those of the upper aerodigestive tract, the liver, the large intestine and the female breast. Multiple mechanisms are involved in alcohol-mediated carcinogenesis. Among those the action of acetaldehyde (AA), the first metabolite of ethanol oxidation is of particular interest. AA is toxic, mutagenic and carcinogenic in animal experiments. AA binds to DNA and forms carcinogenic adducts. Direct evidence of the role of AA in alcohol-associated carcinogenesis derived from genetic linkage studies in alcoholics. Polymorphisms or mutations of genes coding for AA generation or detoxifying enzymes resulting in elevated AA concentrations are associated with increased cancer risk. Approximately $40 \%$ of Japanese, Koreans or Chinese carry the AA dehydrogenase $2 * 2($ ALDH2 2 ) allele in its heterozygous form. This allele codes for an ALDH2 enzyme with little activity leading to high AA concentrations after the consumption of even small amounts of alcohol. When individuals with this allele consume ethanol chronically, a significant increased risk for upper alimentary tract and colorectal cancer is noted. In Caucasians, alcohol dehydrogenase $1 C^{*} 1$ (ADH1C*1)
\end{abstract}

H. K. Seitz

Department of Medicine, Salem Medical Center,

Heidelberg, Germany

H. K. Seitz $(\bowtie)$

Center of Alcohol Research, Liver Disease and Nutrition,

Heidelberg, Germany

e-mail: helmut_karl.seitz@urz.uni-heidelberg.de

F. Stickel

Institute of Clinical Pharmacology, University of Bern,

Bern, Switzerland allele encodes for an $\mathrm{ADH}$ isoenzyme which produces 2.5 times more $\mathrm{AA}$ than the corresponding allele $\mathrm{ADH} 1 \mathrm{C} * 2$. In studies with moderate to high alcohol intake, ADH1C*1 allele frequency and rate of homozygosity was found to be significantly associated with an increased risk for cancer of the upper aerodigestive tract, the liver, the colon and the female breast. These studies underline the important role of acetaldehyde in ethanol-mediated carcinogenesis.

Keywords Alcohol · Acetaldehyde .

Alcohol dehydrogenase - Upper aerodigestive tract cancer

\section{Introduction}

Although alcoholism is a frequently observed disease and although chronic heavy alcohol consumption damages almost every tissue and organ of the human body, the occurrence of certain alcohol-associated organ injuries is rather low. Only $10-15 \%$ of heavy drinkers develop, for example, liver cirrhosis and an even smaller proportion of drinkers develop cancer. However, chronic alcohol consumption is a risk factor for certain types of cancer including the upper aerodigestive tract, the liver, the colorectum, and the breast [1]. Worldwide, a total of approximately 389,000 cases of cancer representing $3.6 \%$ of all cancers (5.2\% in men and $1.7 \%$ in women) derive from chronic alcohol consumption [2]. As the amount of alcohol is obviously not the only determinant for organ injury, genetic and environmental factors may modulate and determine organ damage or carcinogenesis. A variety of mechanisms may contribute to alcohol-mediated carcinogenesis including the toxic effect of acetaldehyde (AA) and/or reactive oxygen species, disturbed methyl transfer and abnormal metabolism of vitamin A and its derivative 
retinoic acid [3]. In addition, local effects such as cirrhosis of the liver may further stimulate cancer development [3].

Animal experiments have clearly shown that AA, the first metabolite of ethanol oxidation, is carcinogenic $[4,5]$. The concentration of AA in different tissues depends on the production and degradation of AA. Both metabolic steps are modulated by the activity of the ethanol metabolizing enzymes alcohol dehydrogenase $(\mathrm{ADH})$ and acetaldehyde dehydrogenase (ALDH). Genes coding for these enzymes harbor polymorphisms which result in different enzyme activities and, subsequently, to different AA concentrations. In this review, the role of $\mathrm{AA}$ in carcinogenesis and genetic factors modulating AA levels will be discussed in detail. With respect to other factors involved in alcoholmediated carcinogenesis, it is referred to a most recent review article [3].

\section{Toxic and carcinogenic effects of acetaldehyde}

Acetaldehyde is highly toxic, mutagenic and carcinogenic. AA interferes at many sites with DNA synthesis and repair and can, consequently, result in tumor development [6, 7]. Numerous in vitro and in vivo experiments in prokaryotic and eukaryotic cell cultures as well as in animal models have shown that AA has direct mutagenic and carcinogenic effects. It causes point mutations in the hypoxanthineguanine-phosphoribosyl transferase locus in human lymphocytes, induces sister chromatid exchanges, and gross chromosomal aberrations [8-13]. It induces inflammation and metaplasia of tracheal epithelium and enhances cell injury associated with hyperregeneration in the esophageal and colorectal mucosa [14-16]. Thus, when AA was administered in drinking water to rodents [15], the mucosa lesions of the upper aerodigestive tract observed resembled those following chronic alcohol ingestion [16]. Also it has been shown that AA interferes with the DNA repair machinery. AA directly inhibits O6 methyl-guanyltransferase, an enzyme important for the repair of adducts caused by alkylating agents [17].

Moreover, when inhaled, AA causes nasopharyngeal and laryngeal carcinoma [4, 5]. AA also binds rapidly to cellular proteins and DNA which results in morphological and functional impairment of the cell and in an immunologic cascade reaction. The binding to DNA and the formation of stable adducts represent one mechanism by which AA could trigger the occurrence of replication errors and/or mutations in oncogenes or tumor suppressor genes [18]. The occurrences of stable DNA adducts has been shown in different organs of alcohol-fed rodents and in leukocytes of alcoholics [19]. In addition, it has been shown that the major stable DNA adduct, $\mathrm{N}^{2}$-ethyl-2deoxyguanosine $\left(\mathrm{N}^{2}-\mathrm{Et}-\mathrm{dG}\right)$ can indeed be used efficiently by eukaryotic DNA polymerase [20]. While the formation of $\mathrm{N}^{2}$-Et-dG has been shown in DNA samples from white blood cells of human alcoholics and in the liver of rats given ethanol in the drinking water, there is relatively little evidence that this lesion is mutagenic, and the biological significance of the lesion is unclear. However, this lesion can be detected in human urine samples, suggesting that it may be useful as a biomarker of AA-related DNA damage [20]. More recent data have shown that in the presence of basic amino acids or histones, AA reacts with deoxyguanosine in DNA to form another DNA adduct, $1, \mathrm{~N}^{2}$-propano-dG (PdG) [21, 22]. In contrast to $\mathrm{N}^{2}$-Et-dG, PdG has been shown to be a mutagenic DNA lesion in mammalian cells in vivo. These AA-associated effects occurred at AA concentrations from 40 to $1,000 \mu \mathrm{M}$. According to the International Agency for Research on Cancer (IARC) there is sufficient evidence to identify AA as a carcinogen in experimental animals $[6,7]$.

\section{Mechanisms of elevated tissue levels of acetaldehyde}

Production of acetaldehyde by ADH and cytochrome P4502E1

Acetaldehyde is produced from ethanol by $\mathrm{ADH}$ or cytochrome P4502E1 (CYP2E1). Seven isoenzymes for ADH exist, and almost every tissue contains ADH activity. $\mathrm{ADH} 2$ is only present in the liver and ADH4 only in the upper gastrointestinal mucosa [23]. ADH1B and ADH1C are polymorphic and thus code for enzymes capable to produce different amounts of AA [24]. In non-drinkers, CYP2E1, also termed the microsomal ethanol oxidizing system (MEOS), produces only small amounts of AA. However, in chronic alcoholics, CYP2E1 is induced and contributes up to $30 \%$ of the overall ethanol metabolism [25]. This CYP2E1-dependent MEOS also produces reactive oxygen species (ROS) which may be important in alcohol-associated carcinogenesis [26, 27]. In addition, CYP2E1 activates various procarcinogens present in diets and tobacco smoke to their ultimate carcinogens [28]. For more details the recent review articles can be referred $[3,29]$.

Production of acetaldehyde by bacteria

Acetaldehyde is also produced from ethanol by bacterial oxidation in the upper aerodigestive tract and in the large intestine [30-32]. It has been shown that many microbes as part of the normal oral microflora show ADH activity with a variety of individual kinetic characteristics. Under aerobic or microaerobic conditions AA is produced. Thus, AA concentrations between 50 and $150 \mu \mathrm{M}$ can be detected in 
the saliva of individuals after ingestion of moderate dose of ethanol ( $0.5 \mathrm{~g}$ per $\mathrm{kg}$ body weight) [33]. This salivary AA is significantly reduced following the administration of an antiseptic mouthwash demonstrating the importance of microbes in the production of AA from ethanol [33].

Similarly fecal bacteria are also capable to metabolize ethanol to AA [31]. High AA levels have been found after alcohol administration in the colon of rats and these concentrations of AA were significantly lower in germfree animals as compared to conventional rats suggesting that fecal bacteria are capable to produce AA [34]. Indeed, the reversed microbial $\mathrm{ADH}$ reaction produces under aerobic or microaerobic conditions striking amounts of AA when human colonic contents or some microbes representing normal colonic flora are incubated in vitro at $37^{\circ} \mathrm{C}$ with increasing ethanol concentrations [34-36]. This reaction is active already at comparatively low ethanol concentrations (10-100 mg\%), which exist in the colon following social drinking [37]. AA formation catalyzed by microbial $\mathrm{ADH}$ takes place at a $\mathrm{pH}$ normally found in the colon and is rapidly reduced with decreasing $\mathrm{pH}$ [35].

Antibiotics have been used to study the role of colonic bacteria in the oxidation of ethanol to AA. The reduction of aerobic gastrointestinal flora with ciprofloxacin decreases total ethanol elimination by a rate of about $10 \%$ both in rats and in man [38-40]. This is associated with a significant decrease in the mean ADH activity of fecal samples, in almost total abolishment of the formation of endogenous ethanol in the colon, and in a remarkable reduction of the intracolonic AA production from ethanol [39, 40].

Decreased acetaldehyde metabolism by inactive acetaldehyde dehydrogenase

Increased AA concentrations may also occur when its detoxification is inadequate. AA is metabolized by ALDHs [41]. The most important ALDH is ALDH2 with a high affinity to AA mutations of ALDH2 as seen in more than $40 \%$ of Asians result in elevated serum AA concentrations and cancer [42] (Fig. 1).

\section{Role of ADH in alcohol-associated carcinogenesis}

Genetic linkage studies with alcoholics have provided strong support for the assumption that AA plays a central role in alcohol-associated carcinogenesis. These studies found that individuals who accumulate AA because they carry certain alleles of the genes encoding ADH or ALDH have an increased cancer risk [42, 43]. As discussed above there are at least seven isozymes of human $\mathrm{ADH}$ that are encoded by seven genes. These isozymes are categorized into five different classes based on structural characteristics. Class I isozymes account for most of the alcohol metabolism.

For both the $A D H 1 B$ and the $A D H 1 C$ genes, several alleles exist that result in differences in the activity of the

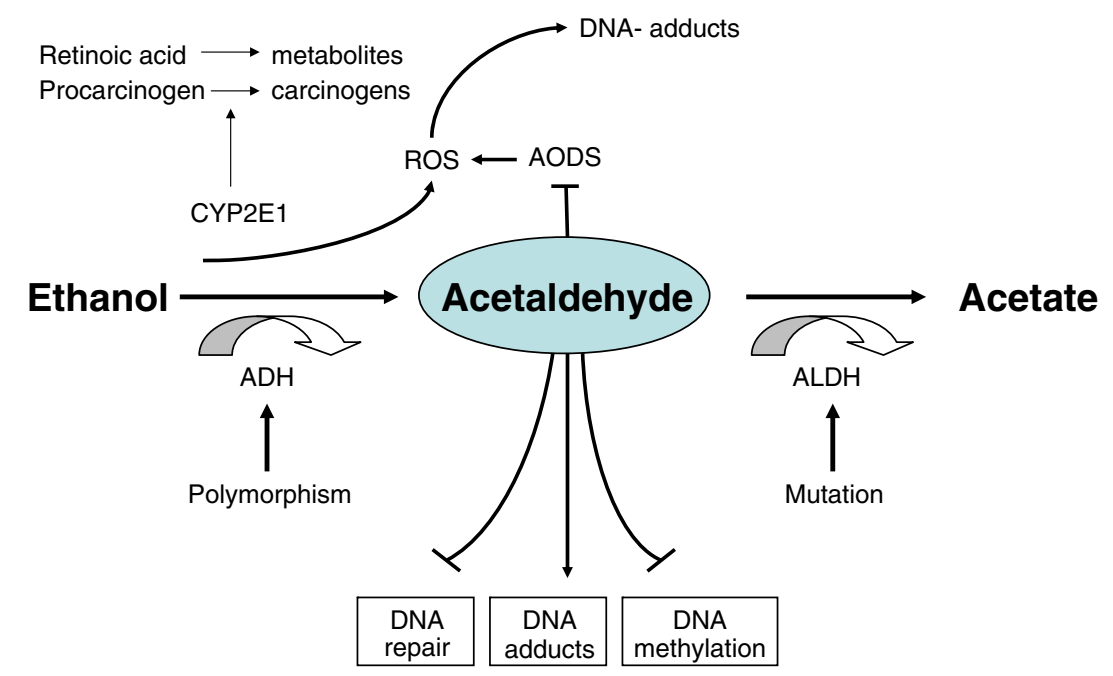

Fig. 1 Ethanol metabolism and its role in carcinogenesis. Ethanol is metabolised via alcohol dehydrogenase $(A D H)$ and via cytochrome P4502E1 (CYP2E1) to acetaldehyde. Acetaldehyde is further metabolised via acetaldehyde dehydrogenase $(A L D H)$ to untoxic acetate. $\mathrm{ADH} 1 \mathrm{~B}$ and ADH1C show polymorphism with different enzyme kinetics and thus different acetaldehyde production. ALDH2 is mutated in Asians resulting in low enzyme activity and accumulation of acetaldehyde. Acetaldehyde is toxic, mutagenic and carcinogenic, results in DNA adducts, inhibits DNA repair and DNA methylation and damages the antioxidative defense system (AODS). In addition, ethanol oxidation via CYP2E1 also generates reactive oxygene species (ROS) which cannot be sufficiently detoxified due to an injured AODS and thus results in DNA adducts. CYP2E1 also activates various procarcinogens to their ultimative carcinogenic metabolites and metabolises retinoic acid resulting in low levels of hepatic retinoic acid 
ADH molecules they encode. For example, the $A D H 1 B * 2$ allele encodes an enzyme that is approximately 40 times more active than the enzyme encoded by the $A D H 1 B^{*} 1$ allele. Similarly, the enzyme encoded by the $A D H I C * 1$ allele is 2.5 times more active than the enzyme encoded by the $A D H I C * 2$ allele [44]. Individuals who carry the highly active $A D H 1 B * 2$ allele rapidly convert ethanol to AA. This leads to AA accumulation following alcohol consumption and results in toxic side effects, such as a flushing syndrome with sweating, accelerated heart rate, nausea, and vomiting. These adverse symptoms exert a protective effect against acute and chronic alcohol consumption (i.e., individuals with this allele typically drink little or no alcohol) and also appear to protect against alcohol-associated cancer development. The $A D H 1 B^{* 2}$ allele is rarely found in Caucasians but occurs more frequently in Asian populations.

It has recently been demonstrated that also the low activity $\mathrm{ADH} 1 \mathrm{~B} * 1 / 1^{*}$ genotype associates with enhanced exposure to AA through saliva. This might be due to lower systemic elimination rate of ethanol from the body which results in prolonged exposure to acetaldehyde produced by oral microbes [45]. There is also strong evidence that the combination of ALDH2-deficiency and slow ADH1B associate with highest risk for esophageal cancer especially among heavy drinkers [46, 47].

The effects of the different $A D H I C$ alleles on alcohol metabolism and, consequently, on drinking levels and alcohol-related carcinogenesis, are more subtle. They can best be studied in Caucasian populations in which the highly active $A D H 1 B * 2$ allele is rare. Studies on the relationship between $A D H 1 C$ alleles and cancer occurrence in Caucasians have led to in contradictory results [48-62]. Harty and colleagues [49] compared the risk of oral cancer associated with various alcohol consumption levels in individuals homozygous for the more active $A D H 1 C^{*} 1$ allele with the risk in heterozygotes who carried only one copy of this allele or were homozygous for the less active $A D H 1 C * 2$ allele. The study found that individuals who consumed eight or more drinks per day and were homozygous for the more active $A D H 1 C^{*} 1$ allele had a 40 -fold increased risk for oral cancer compared with nondrinkers. In contrast, people who consumed the same amount of alcohol but who were heterozygous or homozygous for the less active $A D H 1 C^{*}$ allele had only four- to seven-fold increased risk compared with nondrinkers. At lower levels of alcohol consumption, the difference in cancer risk between the various gene carriers was less striking. This is not surprising, however, because higher levels of alcohol consumption also result in production of more AA which then can exert its carcinogenic effect.

Additional studies have confirmed an increased risk of oropharyngeal and laryngeal cancer in alcohol consumers with the $A D H 1 C^{*} l$ allele $[50,56,60]$. Other case-control studies, however, have not been able to confirm this association [51-55]. The negative results of these studies may, at least in part, result from the fact that the alcohol intake of the participants was low and may not have led to sufficiently high AA levels.

In addition, a similar risk between $A D H 1 C^{*} 1$ homozygosity and breast cancer has been found [57-59].

More recently, we determined $A D H 1 C$ polymorphisms in more than 400 heavy drinkers with daily alcohol intake of more than $60 \mathrm{~g}$ and various cancers of the upper aerodigestive tract, liver, and breast. Cases of cancer patients were compared with carefully matched control patients with alcohol-related diseases (e.g., cirrhosis of the liver, pancreatitis, and alcohol dependence) but no cancer [56, 60]. Cancer patients and control subjects were of similar age and had similar histories of alcohol consumption and cigarette smoking. In this study, significantly more patients with alcohol-related cancers either had at least one $A D H 1 C^{*} 1$ allele, or were homozygous for $A D H 1 C^{*} 1$, than did patients with other alcohol-related diseases. Statistical analyses determined a significant association between $A D H 1 C^{*} l$ allele frequency and rate of homozygosity and an increased risk for alcohol-related cancer $(P<0.001)$. Finally, individuals homozygous for $A D H 1 C^{*} l$ had a relative risk of developing esophageal, liver, and head and neck cancers of 2.9, 3.6, as well as 2.2 , respectively, compared with people homozygous for $A D H 1 C * 2$.

We also found that individuals who are homozygous for the $A D H 1 C^{*} l$ allele had significantly higher AA levels in their saliva than did heterozygous individuals or individuals who are homozygous for the $A D H 1 C * 2$ allele [56]. As mentioned earlier, AA levels in the saliva may be important for cancer development. Saliva rinses the mucosa of the upper aerodigestive tract and any AA in the saliva may be taken up by mucosal cells. Moreover, mucosal cells display little ALDH2 enzyme activity and therefore cannot efficiently detoxify AA. As a result, AA may bind to proteins and DNA in the mucosal cells and may initiate carcinogenesis. The hypothesis that AA in the saliva contributes to tumor development is supported by the observation that AA-fed rats with intact salivary glands showed excessive proliferation of the upper gastrointestinal mucosa [15], similar to the changes observed following chronic alcohol consumption [16, 63, 64]. When the glands were surgically removed (i.e., when the animals no longer produced saliva), however, this excessive cell proliferation disappeared [63].

Because the $\gamma$-ADH enzyme, which is encoded by $A D H I C$, also is found in the mucosa lining the colon, other investigators have studied the relationship between the various $A D H 1 C$ alleles and the development of alcoholrelated colorectal cancer. Tiemersma and co-workers [61] 
have suggested that the $A D H I C * 1$ allele can play an important role in the development of alcohol-associated colon cancer. To evaluate whether the association between alcohol consumption and colorectal tumor development is modified by $A D H 1 C$ polymorphism, we recruited 173 individuals with colorectal tumors diagnosed by colonoscopy and 788 control individuals without colorectal tumors [62]. Genotype $A D H 1 C^{*} 1 / 1$ was more frequent in patients with alcohol-associated colorectal neoplasia compared to patients without cancers (OR 1.67, $P=0.014$ ) [51]. Furthermore, we could show that the difference in $A D H 1 C$ polymorphism between controls and colorectal neoplasia is strongly influenced by alcohol consumption and that individuals with more than $30 \mathrm{~g}$ alcohol per day with the genotype $A D H 1 C^{*} 1 / 1$ had an increased risk for colorectal cancer [62].

In summary, numerous studies suggest that $A D H 1 C$ alleles that result in AA accumulation in the cells can enhance a drinker's risk of developing alcohol-related cancers in a variety of tissues. An important prerequisite for this mechanism is, however, that the amount of alcohol consumed is high enough to generate AA concentrations at a mutagenic level $(>60 \mu \mathrm{M})$.

\section{Role of ALDH in alcohol-associated carcinogenesis}

The main enzyme that breaks down AA is ALDH2. It is encoded by the $A L D H 2$ gene, for which there are two main alleles, $A L D H 2 * 1$ and $A L D H 2 * 2$. The $A L D H 2 * 2$ allele differs from the normal $A L D H 2 * 1$ allele by an nucleotide substitution $(\mathrm{G}>\mathrm{A})$ in exon 12 of the $A L D H 2$ gene, resulting in an almost inactive ALDH enzyme. This allele does not occur in Caucasians and is only found among Asians. For example, approximately $10 \%$ of the Japanese population are homozygous for $A L D H 2 * 2$. Moreover, approximately $40 \%$ of the Asian population are heterozygous. People who are homozygous for $A L D H 2 * 2$ have an extremely low ALDH activity; when these people drink alcohol, AA accumulates and the "flushing syndrome" develops [42, 44]. These subjects do not tolerate alcohol at all and are, therefore, generally protected against developing alcoholism. Those who are heterozygous also have greatly reduced (i.e., less than 10\%) ALDH2 activity. Nevertheless, they tolerate alcohol ingestion and may even become heavy drinkers and alcoholics.

Several epidemiological studies have demonstrated that the risk of alcohol-associated cancer of the aerodigestive tract is significantly elevated in people with low ALDH2 activity, with a relative risk of 11.0 for oropharyngeal and laryngeal cancer and 12.5 for esophageal cancer [42, 43, $65,66]$.
In addition, these people have a 50-fold higher risk than people without the $A L D H 2 * 2$ allele of simultaneously developing a second tumor at another site of the esophagus. Finally, the risk of colon cancer is increased by a factor of 3.4 in individuals with an $A L D H 2 * 2$ allele [43].

As in the case with individuals who are homozygous for the highly active $A D H 1 C^{*} 1$ allele, individuals who have one $A L D H 2 * 2$ allele have elevated AA levels in their saliva after a moderate dose of alcohol [67]. In fact, AA levels are nine times higher in the saliva than in the blood of these people, suggesting that it is reduced ALDH activity in the salivary glands rather than in the blood that leads to AA accumulation in the saliva. Whenever they drink, people of this group are exposed to extremely high AA concentrations in their saliva, which is associated with a strikingly increased cancer risk. The harmful effects of salivary AA are exacerbated further by the previously mentioned fact that AA can be converted to cancer-causing crotonaldehyde in the presence of polyamines [21, 22], which are elevated in tissue already injured by the local action of alcohol, such as the mucosa of the upper aerodigestive tract [16].

It is worth noting that on the basis of these Japanese studies, the International Agency for Research on Cancer (IARC) concluded that AA has a causal role in ethanolrelated esophageal carcinogenesis [1].

\section{Summary and conclusions}

Chronic alcohol consumption is a risk factor for cancer of the oropharynx, hypopharynx, larynx, esophagus, liver, colorectum and breast. Various mechanisms contribute at the molecular level to this cancer risk. Among others AA generated from ethanol via $\mathrm{ADH}, \mathrm{CYP} 2 \mathrm{E} 1$ or by gastrointestinal bacteria stimulates carcinogenesis and also binds to DNA forming mutagenic and carcinogenic DNA adducts. Evidence comes from in vitro studies in cell cultures, from animal studies and finally from human genetic linkage studies. Individuals who accumulate AA either due to an enhanced AA production or to a reduced metabolism of AA have such an increased cancer risk for all ethanolspecific organ sites. Japanese with an ineffective AA dehydrogenase activity due to a mutation in the ALDH gene have a striking increased risk for esophageal cancer when they consume alcohol. In addition, Caucasians who produce more AA due to a polymorphism in the ADH1C gene following alcohol ingestion also reveal an increased cancer risk. Prerequisite, however, is the intake of a certain amount of ethanol leading to AA levels high enough to form DNA adducts. These observations in man underline the important role of AA in ethanol-mediated carcinogenesis. 


\section{Future research directions}

Among the many aspects of ethanol-related carcinogenesis, the role of gene-environment interactions deserves particular attention. Although our understanding of the impact of both host and environmental factors on certain diseases has substantially grown, it remains still difficult to clearly separate their influence on an individual's phenotype. As an example, it is yet unclear what precise attributable risk derives from the carriage of certain genetic variants that result in higher AA concentrations as opposed to the presence of other established environmental risk factors, such as smoking or over nutrition. Potential interactions between host and environmental disease modifiers may vary with respect to the organ under scrutiny and could be either additive or synergistic. For upper aerodigestive tract cancer, smoking and poor oral hygiene are such environmental risk factors that amplify the detrimental effects of concomitant alcohol consumption. In the liver, the role of coexisting chronic liver diseases such as hepatitis B and C virus infection, iron storage diseases or steatosis due to non-alcoholic causes (NAFLD) should be investigated in individuals with genetically distinct acetaldehyde burden. Of course, such data would also be usefully for breast and colon cancer. With regard to the latter two diseases the relevance of a family history of cancer, estrogen replacement therapy, or coexisting inflammatory bowel disease on the risk of breast or colorectal cancer in subjects with ADH1C*1/1 and ALDH2*1/2 warrants further research. Obviously, such interactions would greatly impact preventive measures in affected individuals and justify screening colonoscopy or mammography to increase the rates of early detection.

Another important research task issue is how to detoxify AA before it may exert its carcinogenic effect. This may be especially relevant for salivary AA since there is scientific evidence that this can be detoxified through its binding to glutathione or similar reductive equivalents contained in chewing gum or tablets. Such an approach could be also used as a preventive intervention for cancers of the oral cavity or upper aerodigestive tract.

Acknowledgment Original research reviewed in this manuscript has been supported by a grant of the Dietmar Hopp Foundation and the Manfred Lautenschläger Foundation. The authors wish to thank Mrs. A. Erb for typing the manuscript. This manuscript is dedicated to Charles S. Lieber, MD in grateful remembrance.

\section{References}

1. Baan R et al (2007) Carcinogenicity of alcoholic beverages. Lancet Oncol 8:292-293

2. Rehm J et al (2004) Comparative quantification of health risks: global and regional burden of disease attributable to selected major risk factors. In: Ezzati M, Murray C, Lopez AD, Rodgers A (eds) World Health Organization, Geneva, pp 959-1108

3. Seitz HK, Stickel F (2007) Molecular mechanisms of alcoholmediated carcinogenesis. Nat Rev Cancer 7:599-612

4. Woutersen RA, Appelmann LM, Van Garderen-Hoetmer A, Feron VJ (1986) Inhalation toxity of acetaldehyde in rats: III. Carcinogenicity study. Toxicology 41:213-231

5. Feron VJ, Kruysse A, Woutersen RA (1982) Respiratory tract tumours in hamsters exposed to acetaldehyde vapour alone or simultaneously to benzo(a)pyrene or diethylnitrosamine. Eur $\mathbf{J}$ Cancer Clin Oncol 18:13-31

6. Anonymous (1985) Acetaldehyde. IARC Monogr Eval Carcinog Risk Chem Hum 36

7. IARC (2009) Alcoholic beverage consumption and ethyl caramate (urethane). IARC monographs on the evaluation of carcinogenic risks to humans 96 (International Agency for Research on Cancer, Lyon (in press)

8. Dellarco VL (1988) A mutagenicity assessment of acetaldehyde. Mutat Res 195:1-20

9. Helander A, Lindahl-Kiessling K (1991) Increased frequency of acetaldehyde-induced sister-chromatide exchanges in human lymphocytes treated with an aldehyde dehydrogenase inhibitor. Mutat Res 264:103-107

10. Obe G, Jonas R, Schmidt S (1986) Metabolism of ethanol in vitro produces a compound which induces sister-chromatid exchanges in human peripheral lymphocytes in vitro: acetaldehyde not ethanol is mutagenetic. Mutat Res 174:47-51

11. Maffei $F$ et al (2000) Increased cytogenetic damage detected by FISH analysis on micronuclei in peripheral lymphocytes from alcoholics. Mutagenesis 15:517-523

12. Maffei $F$ et al (2002) Biomarkers to assess the genetic damage induced by alcohol abuse in human lymphocytes. Mutat Res 514:49-58

13. Matsuda T, Kawaishi M, Yagi T, Matsui S, Takebe H (1998) Specific tandem GG to TT base substitutions induced by acetaldehyde are due to intra-strand crosslinks between adjacent guanine bases. Nucleic Acids Res 26:1769-1774. A cell culture study demonstrating the evolution of mutations such as interstrand crosslinks in human cells exposed to acetaldehyde

14. Simanowski UA et al (1994) Enhancement of ethanol induced rectal mucosal hyperregeneration with age in F244 rats. Gut 35:1102-1106

15. Homann N, Kärkkäinen P, Koivisto T, Nosova T, Jokelainen K, Salaspuro M (1997) Effects of acetaldehyde on cell regeneration and differentiation of the upper gastrointestinal tract mucosa. $\mathrm{J}$ Natl Cancer Inst 85:1692-1697

16. Simanowski UA et al (1993) Esophageal epithelial hyperregeneration following chronic ethanol ingestion: effect of age and salivary gland function. J Natl Cancer Inst 85:2030-2033

17. Espina N, Lima V, Liba CS, Garro AJ (1988) In vitro and in vivo inhibitory effect of ethanol and acetaldehyde on O6-methylguanine transferase. Carcinogenesis 9:761-766

18. Fang JL, Vaca CE (1995) Development of a 32P-postlabeling method for the analysis of adducts arising through the reaction of acetaldehyde with $2^{\prime}$-deoxyguanosine- $3^{\prime}$-monophosphate and DNA. Carcinogenesis 16:2177-2185

19. Fang JL, Vaca CE (1997) Detection of DNA adducts of acetaldehyde in peripheral white blood cells of alcohol abusers. Carcinogenesis 18:627-632

20. Matsuda T, Terashima I, Matsumoto Y, Yaboshita H, Matsui S, Shibutani S (1999) Effective utilization of N2-ethyl-2'-deoxyguanosine triphosphate during DNA synthesis catalyzed by mammalian replicative DNA polymerases. Biochemistry 38:929935

21. Brooks PJ, Theruvathu JA (2006) Acetaldehyde-DNA adducts: implications for the molecular mechanism of alcohol related 
carcinogenesis. In: Cho CH, Purohit V (eds) Alcohol, tobacco and cancer. Karger, Basel, pp 78-96

22. Theravathu JA, Jaruga P, Nath RG et al (2005) Polyamines stimulate the formation of mutagenic 1, N2-propanodeoxyguanosinase adducts from acetaldehyde. Nucleic Acids Res 33:3513-3520

23. Seitz HK, Oneta CM (1998) Gastrointestinal alcohol dehydrogenases. Nutr Rev 56:52-60

24. Bosron WF, Li TK (1986) Genetic polymorphism of human liver alcohol and aldehyde dehydrogenase and their relationship to alcohol metabolism and alcoholism. Hepatology 6:502-510

25. Lieber CS (1994) Alcohol and the liver 1994 update. Gastroenterology 106:1085

26. Frank A, Seitz HK, Bartsch H, Frank N, Nair J (2004) Immunohistochemical detection of 1, N6-ethenodeoxyadenosine in nuclei of human liver affected by diseases predisposing to hepato carcinogenesis. Carcinogenesis 25:1027-1031

27. Wang Y, Millonig G, Nair J, Patsenker E, Stickel F, Mueller S, Bartsch H, Seitz HK (2009) Ethanol-induced cytochrome P$4502 \mathrm{E} 1$ causes carcinogenic etheno-DNA lesions in alcoholic liver disease. Hepatology (in press)

28. Seitz HK, Oswald B (1992) Effect of ethanol on procarcinogen activation. In: Watson RR (eds) Alcohol and cancer. CRC Press, Boca Raton, pp 55-72

29. Seitz HK, Stickel F (2006) Risk factors and mechanisms of hepatocarcinogenesis with special emphasis on alcohol and oxidative stress. Biol Chem 387:349-360

30. Salaspuro M (2003) Acetaldehyde, microbes, and cancer of the digestive tract. Crit Rev Clin Lab Sci 40:183-208

31. Seitz HK, Pöschl G, Salaspuro M (2006) Alcohol and colorectal cancer. In: Cho CH, Purohit V (eds) Alcohol, tobacco and cancer. Karger, Basel, pp 63-77

32. Salaspuro M, Salaspuro V, Seitz HK (2006) Alcohol and upper aerodigestive tract cancer. In: Cho $\mathrm{CH}$, Purohit V (eds) Alcohol, tobacco and cancer. Karger, Basel, pp 48-62

33. Homan N, Jousimies-Somer H, Jokelainen K, Heine R, Salaspuro M (1997) High acetaldehyde levels in saliva after ethanol consumption: methodogical aspects and pathogenetic implications. Carcinogenesis 18:1739-1743

34. Seitz HK, Simanowski UA, Garzon FT, Rideout JM, Peters TJ, Koch A, berger MR, Einecke H, Maiwald M (1990) Possible role of acetaldehyde in ethanol-related rectal cocarcinogenesis in the rat. Gastroenterology 98:406-413

35. Jokelainen K, Roine R, Väänänen H, Salaspuro M (1994) In vitro acetaldehyde formation by human colonic bacteria. Gut 35:12711274

36. Jokelainen K, Siitonen A, Jousimies-Somer H (1996) In vitro alcohol dehydrogenase-mediated acetaldehyde production by aerobic bacteria representing the normal colonic flora in man. Alcohol Clin Exp Res 20:967-972

37. Salaspuro V, Nyfors S, Heine R, Siitonen A, Salaspuro M, Jousimies-Somer H (1999) Ethanol oxidation and acetaldehyde production in vitro by human intestinal strains of Eschericia coli under aerobic, microaerobic and anaerobic conditions. Scand J Gastroenterol 34:967-973

38. Jokelainen K, Matysiak-Budnik T, Mäkisalo H, Höckerstedt K (1996) Salaspuro M: high intracolonic acetaldehyde values produced by a bacteriocolonic pathway for ethanol oxidation in piglets. Gut 39:100-104

39. Jokelainen K, Nosova T, Koivisto T, Väkeväinen S, JousimiesSomer H, Heine R (1997) Salaspuro M: inhibition of bacteriocolonic pathway for ethanol oxidation by ciprofloxacin in rats. Life Sci 61:1755-1762

40. Tillonen J, Homann N, Rautio M, Jousimies-Somer H, Salaspuro M (1999) Ciprofloxacin decreases the rate of ethanol elimination in humans. Gut 44:347-352
41. Pares X, Farres J (1996) Alcohol and aldehyde dehydrogenases in the gastrointestinal tract. In: Preedy VR, Watson Ronald R (eds) Alcohol and the gastrointestinal tract. CRC Press, Boca Raton

42. Seitz HK, Matsuzaki S, Yokoyama A, Homan N, Vacevainen S, Wang XD (2001) Alcohol and cancer. Alcohol Clin Exp Res 25:137-143

43. Yokoyama A, Muramatsu T, Ohmori T, Yokoyama T, Okuyama K, Takahashi H, Hasegawa Y, Higuchi S, Maruyama K, Shirakura K, Ishiii H (1998) Alcohol-related cancers and aldehydrogenase-2 in Japanese alcoholics. Carcinogenesis 19:13831387

44. Bosron WF, Ehrig T, Li TK (1993) Genetic factors in alcohol metabolism and alcoholism. Semin Liver Dis 13:126-135 PMID: 8337601

45. Yokoyama A, Tsutsumi E, Imazeki H, Suwa Y, Nakamura C, Yokoyama T (2007) Contribution of the alcohol dehydrogenase1B genotype and oral microorganisms to high salivary acetaldehyde concentrations in Japanese alcoholic men. Int $\mathrm{J}$ Cancer 121:1047-1054

46. Lee CH, Lee JM, Wu DC et al (2008) Carcinogenetic impact of ADH1B and ALDH2 genes on squamous cell carcinoma risk of the esophagus with regard to the consumption of alcohol, tobacco and betel quid. Int J Cancer 122:1347-1356

47. Yang SJ, Wang HY, Li XQ et al (2007) Genetic polymorphisms of $\mathrm{ADH} 2$ and $\mathrm{ALDH} 2$ association with esophageal cancer risk in southwest China. World J Gastroenterol 13:5760-5764

48. Brennan E et al (2004) Pooled analyssis of alcohol dehydrogenase genotypes and head and neck cancer: a huge review. Am J Epidemiol 159:1-16

49. Harty LC et al (1997) Alcohol dehydrogenase 3 genotype and risk of oral cavity and pharyngeal cancers. J Natl Cancer Inst 89:1698-1705

50. Coutelle C et al (1997) Laryngeal and oropharyngeal cancer and alcohol dehydrogenase 3 and glutathione S-transferase M1 polymorphism. Hum Genet 99:319-325

51. Olshan AF, Weissler MC, Watson MA, Da Bell (2001) Risk of head and neck cancer and the alcohol dehydrogenase 3 genotype. Carcinogenesis 22:57-61

52. Sturgis EM et al (2001) Alcohol dehydrogenase 3 genotype is not associated with risk of squamous cell carcinoma of the oral cavity and pharynx. Cancer Epidemiol Biomarkers Prev 10:273-275

53. Schwartz SM, Doody DR, Fritzgibbon ED, Ricks S, Porter PL, Chen (2001) Oral squamous cell cancer risk inrelation to alcohol consumption and alcohol dehydrogenase 3 genotypes. Cancer Epidemiol Biomarkers Prev 10:1137-1144

54. Zavras AI et al (2002) Interaction between a single nucleotide polymorphism in the alcohol dehydrogenase 3 gene, alcohol consumption and oral cancer risk. Int J Cancer 97:526-530

55. Risch A et al (2003) Laryngeal cancer risk in Caucasians is associated with alcohol and tobacco consumption but not modified by genetic polymorphism in class 1 alcohol dehydrogenases ADH1B and ADH1C and gluthathione-S-trasferases GSTM1 and GSTT 1. Pharmacogenetics 13:225-230

56. Visapää JP et al (2004) Increased cancer risk in heavy drinkers with the alcohol dehydrogenase $3 * 1$-allel possibly due tom the salivary acetaldehyde. Gut 53:871-876

57. Coutelle C, Höhn B, Benesova M et al (2004) Risk factors in alcohol-associated breast cancer: alcohol dehydrogenase polymorphism and estrogens. Int J Oncol 25:1127-1132

58. Freudenheim JL, Ambrosone CB, Moysich KB et al (1999) Alcohol dehydrogenase 3 genotype modification of the association of alcohol consumption with breast cancer risk. Cancer Causes Control 10:369-377

59. Terry MB, Gammon MD, Zhang FF et al (2006) ADH3 genotype, alcohol intake and breast cancer risk. Carcinogenesis 27(4):840847 
60. Homann N, Stickel F, König IR et al (2006) Alcohol dehydrogenase $1 \mathrm{C}^{*} 1$ allele is a genetic marker for alcohol-associated cancer in heavy drinkers. Int J Cancer 118:1998-2002

61. Tiemersma EW, Wark PA, Ocke MC et al (2003) Alcohol consumption, alcohol dehydrogenase 3 polymorphism, and colorectal adenomas. Cancer Epidemiol Biomarkers Prev 12:419-425

62. Homann N, König I, Marks M, Benesova M, Stickel F, Millonig G, Mueller S, Seitz HK (2009) Alcohol and colorectal cancer: role of alcohol dehydrogenase 1C polymorphism. Alcoholism Clin Exp Res 33(3):551-565

63. Maier H, Born IA, Veith S, Adler D, Seitz HK (1986) The effect of chronic ethanol consumption on salivary gland morphology and function in the rat. Alcohol Clin Res 10:425-427
64. Maier H et al (1994) Effect of chronic alcohol consumption on the morphology of the oral mucosa. Alcohol Clin Res 18:387-391

65. Yokoyama A et al (1996) Multiple primary esophageal and concurrent aerodigestive tract cancer and the aldehyde dehydrogenase-2 genotype of Japanese alcoholics. Cancer 77:1986-1990

66. Yokoyama A et al (1999) Alcohol and aldehyde gene polymophisms influence suspectibility to esophageal cancer in Japanese alcoholics. Alcohol Clin Exp Res 23:1705-1710

67. Väkeväinen S, Tillonen J, Agawal D, Srivastava N, Salaspuro M (2000) High salivary acetaldehyde after a moderate dose of alcohol in ALDH2-deficient subjects: strong evidence for the local carcinogenic action of acetaldehyde. Alcohol Clin Exp Res 24(6):873-877 